\title{
Improving the models for diffraction used in serial crystallographic data reduction
}

Aaron S. Brewster ${ }^{a}$, Iris D. Young ${ }^{a}$, and Nicholas K. Sauter ${ }^{a}$

${ }^{a}$ Lawrence Berkeley Laboratory, Berkeley, CA 94720

Serial crystallography is an expanding field at XFELs and at synchrotrons, but serious challenges remain for data processing pipelines. Crystals are typically not rotated in the beam generating 'still shots' that represent only a slice of reciprocal space. With fewer reflections to determine unit cell parameters and crystal orientation, and without a direct way to measure mosaic parameters from a rocking curve, predicting which pixels contain signal is difficult. Integration of single pixel reflections at high resolution has required improvements in the accuracy and precision of models used in still shot crystallography, and these improvements are presented here, as implemented in cctbx.xfel and DIALS. 